\title{
2D Mixture Fraction Studies In A Hot-jet Ignition Configuration Using NO-LIF and Correlation Analysis
}

\author{
Rajesh Sadanandan ${ }^{1}$, Detlef Markus ${ }^{1}$, Robert Schießl ${ }^{2}$, Ulrich Maas ${ }^{2}$ \\ ${ }^{1}$ Physikalisch-Technische Bundesanstalt, Braunschweig, Germany \\ ${ }^{2}$ Institute of Technical Thermodynamics, University of Karlsruhe, Germany
}

\section{Introduction}

Ignition of fuel/air mixtures by hot turbulent gas jets is very important for the combustion community in general e.g. in view of its application in the field of nuclear safety, pulse detonation engines, or supersonic combustors. This work investigates the fundamental processes behind hot-jet ignition by measurements of mixture fraction in a simple hot-jet configuration. Mixture fraction is measured by means of laserinduced fluorescence (LIF) of nitric oxide seeded to the flow. Since the LIF-signal is influenced by many different quantities (temperature, overall chemical composition), and not by the mixture fraction alone, additional information is taken from representative numerical simulations in order to transfer LIF-signals into mixture fraction values. The method exploits the existence of many sharp correlations between different quantities (temperature, species concentrations). The existence of a large number of correlations is empirically obvious from numerical simulations, and theoretically guaranteed by the results of mathematical analyses (like, e.g., Intrinsic Low Dimensional Manifolds (ILDM) [1]).

\section{Methodology}

In order to investigate the hot-jet ignition, Laser Schlieren imaging was employed along with planar NO-LIF to study the spatial and temporal development of the turbulent jet for mixture fraction studies. For the evaluation of the LIF-signals, a method based on reduced state spaces was employed. These approaches will be explained in the next sections.

\subsection{Experimental Setup}

A flash lamp-pumped, frequency tripled Nd:YAG laser is used for pumping a tuneable dye laser (line width $0.002 \mathrm{~nm}$ ). Based on the signal evaluation the frequency doubled output from the dye laser is tuned to coincide with the $\mathrm{Q}_{1}(23.5)$ transition in the $v^{\prime \prime}=0, v^{\prime}=0$ band of the $A^{2} \Sigma^{+}-X^{2} \Pi$ system of NO near $226 \mathrm{~nm}$. The laser beam (pulse energy $1.2 \mathrm{~mJ}$, pulse duration $\approx 8 \mathrm{~ns}$ ) is formed into a sheet of approximately $20 \mathrm{~mm}$ in height and $300 \mu \mathrm{m}$ in thickness by means of cylindrical lenses. Subsequent NO-LIF fluorescence was observed using a Schott UG5 filter. A small portion of the laser sheet is split off and focused into a quartz glass cuvette containing a diluted solution of fluorescent dye. The fluorescence emerging out of the cuvette was imaged onto the side of the same CCD as used for NO-PLIF. This image was used to compensate for shot-to-shot variations in the laser sheet profile. 
The combustion cell used for the NO-PLIF measurements is a simple and well-defined optically accessible constant-volume cell [2]. It consists of two vessels with the first vessel flanged to the second chamber. The two vessels are interconnected by means of a constant diameter nozzle of length $25 \mathrm{~mm}$. In this work nozzles in the diameter range from $0.7 \mathrm{~mm}$ up to $1.2 \mathrm{~mm}$ are examined. The cylindrical small vessel is $80 \mathrm{~mm}$ in length and $60 \mathrm{~mm}$ in diameter. The second vessel has a volume of 12 litres and is provided with three quartz windows for optical access. At the beginning of each experiment both the vessels are filled with hydrogen/air mixture $(\phi=0.95)$. The fuel/air mixture in the first vessel is seeded with $1500 \mathrm{ppm}$ of NO and is ignited by means of electrical discharges on the symmetrical axis of the vessel at a distance $X_{i}$ from the inlet of the nozzle. The hot burned gas from the first vessel expands through the nozzle rendering a jet of hot burned gases that impinges into the unburned hydrogen/air mixture in the second vessel possibly initiating ignition and combustion. The Schlieren and NO-PLIF images are captured along the line of sight and at right angles to the expanding jet flow respectively by means of ICCD cameras. The imaged region is $30 \times 40 \mathrm{~mm}$ (height, width) and has a spatial resolution of approximately $100 \mu \mathrm{m}$. The experiments were repeated with different $X_{i}$ values leading to different pressure ratios $p_{1} / p_{2}$ across the nozzle and therefore to different ignition behaviour in the hot jet flow.

\subsection{Signal evaluation based on reduced state spaces}

To infer mixture fractions from NO-LIF images is not straightforward, because the measured LIF-signal intensity $I_{\exp }$ is influenced not only by the amount of NO molecules but also by effects like quenching, pressure broadening of the absorption lines, which in turn depends on pressure, temperature and chemical composition.

At any location $\boldsymbol{r} \equiv\left(r_{x}, r_{y}, r_{z}\right)$ and time $t$, the instantaneous state of a reacting flow can be expressed by the values of temperature $T(\boldsymbol{r}, t)$, pressure $p(\boldsymbol{r}, t)$ and species mole fractions $x_{i}(\boldsymbol{r}, t)$, with $i=1, \ldots, n_{s}$ and $n_{s}$ as the total number of chemical species. These quantities (state variables) can be arranged into a state vector $\boldsymbol{\psi} \equiv\left(T, p, x_{i}\right)$.

The complex dependency of $I_{\text {exp }}$ on temperature, pressure and chemical composition emitted from a small volume can formally be written as a function of these variables and experimental parameters

$$
I_{\exp }=f\left(p, T, x_{i} ; \boldsymbol{a}\right),
$$

where the vector $\boldsymbol{a}$ represents the experimental parameters. Eq. 1 represents one equation for $n_{s}+2$ unknowns, i.e. it is an underdetermined equation system. It will therefore in general not be possible to determine a state variable, if no additional information is available. We therefore seek for more equations that we can add to eq. (1), so that a fully closed equation system (with $n_{s}+2$ equations) results. In this work, correlations between the state variables are identified indirectly by analyzing the results from a large set of numerical simulations. These simulations are performed to "mimick" the behavior of a hot exhaust gas jet/fresh hydrogen/air mixture. The simulations are one-dimensional unsteady flamelet simulations, which are employed to assess which possible combinations of state variables can occur during the reactive mixing of hot exhaust gas with unburned hydrogen/air mixture. The boundary conditions of the flamelet simulations are selected so that they mimick the situation in the hot jet according to the experiment described above. On the left boundary there is exhaust gas (identified by index (b)) according to chemical equilibrium of a hydrogen/air mixture $(\phi=0.95)$ at $p=1$ bar, $T=T^{(\mathrm{b})}$. Multiple simulations were performed, with values of $T^{(\mathrm{b})}$ varying in the range $700 \mathrm{~K} \ldots 1400 \mathrm{~K}$, and the mole fractions $x_{i}^{(\mathrm{b})}$ set to the corresponding equilibrium compositions. The right side (superscript (u) ) was intially set to unburned $\mathrm{H}_{2}$ /air mixture with $\phi=0.95, p=1$ bar, $T^{(\mathrm{u})}=300 \mathrm{~K}$. For solving the unsteady behavior of the system, the values at the left boundary were kept fixed at the initial values, while at the right boundary, the spatial gradients of all scalars were set to zero.

The simulations include the underlying physical and chemical processes during the mixing of hot burned gases with an unburned hydrogen/air mixture, like detailed chemistry, and detailed transport. The underlying kinetic system for the simulations is a $\mathrm{H}_{2} / \mathrm{O}_{2} / \mathrm{NO}$ reaction mechanism with 19 species and 158 reactions, which is part of a detailed mechanism for the modelling of NO-formation in hydrocarbon 


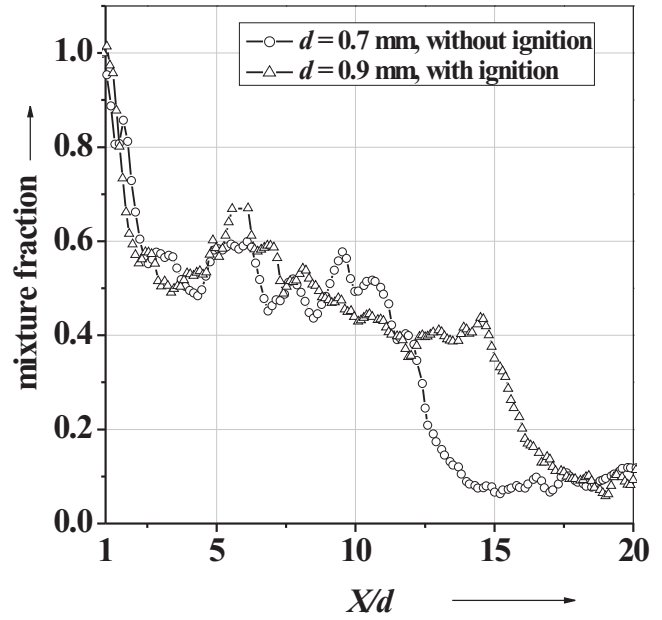

(a)

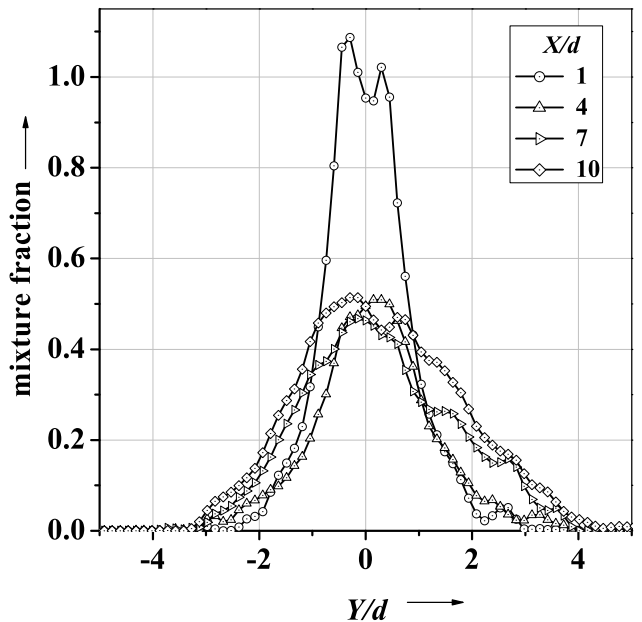

(b)

Figure 1: (a) Axial profiles and (b) radial profiles deduced from the 2D mixture fraction maps $\left(d=0.7 \mathrm{~mm}, p_{1} / p_{2}=1.5\right)$

flames [3]. The information so obtained is then used for the spectroscopic simulation of the NO LIF signal as a function of these state variables using the program LIFSim [4]. We receive a large set (around 50000) of computed state vectors $\psi$, which are "sampled" from the unsteady flamelet simulation.

If we define the mixture fraction in our case as fractional part of the mass originating from the hot gas stream, and if we assume that $Y_{\mathrm{NO}}$ is a conserved scalar, we can define a mixture fraction $\xi_{\mathrm{NO}}$ based on the local NO mass fraction $Y_{\mathrm{NO}}[5]$

$$
\xi_{\mathrm{NO}}=\frac{Y_{\mathrm{NO}}-Y_{\mathrm{NO}}^{(\mathrm{u})}}{Y_{\mathrm{NO}}^{(\mathrm{b})}-Y_{\mathrm{NO}}^{(\mathrm{u})}}
$$

Here, $Y_{\mathrm{NO}}^{(\mathrm{b})}$ and $Y_{\mathrm{NO}}^{(\mathrm{u})}$ are the mass fractions of NO at the hot gas and fresh gas side, respectively. It is then checked if there exist correlations between $\xi$ and $I$ of the form $\xi=g(I)$, with some function $g$. If so, this function is used later to transform measured signals $I_{\exp }$ into mixture fractions. A part of the strategy is to search for LIF-excitation schemes that lead to sharp correlations between the LIF signal and NO mass fraction. These correlations describe a dependence of the LIF signal on the mixture fraction, which is valid for the conditions covered by the underlying set of numerical simulations. Experiments are then performed using this LIF excitation scheme and the measured planar LIF signals are converted back into two-dimensional and spatially resolved NO mixture fraction maps using the correlations.

\section{Results}

Figure 1 shows the instantaneous axial and radial profiles inside hot jet flows deduced from the mixture fraction profiles. The method is not applicable for $X / d<1$ due to interference by laser light scattered from the nozzle. In all the instantaneous profiles the mixture fraction fluctuations arising from the flow turbulence were visible. The axial profile shows a rapid decrease with downstream distance, which is indicative of the hot jet/unburned gas mixing. Due to the experimental method there is a small amount of NO in the outflowing cold gas in front of the hot jet, which leads to an error in the calculation of the mixture fraction in the area. This is visible in Figure 1 at $X / d>12$. The progressive decay from 
the center value of the jet to the border as seen in the radial profiles in Figure 1(b) shows the rapid entrainment and turbulent mixing of the cold unburned mixture with the hot gas of the jet due to strong shear induced turbulence. This highlights the different driving forces behind mixing in the axial and radial direction. Also noticeable in the radial profiles are mixture fraction values slightly exceeding the value one, which is an artefact. The main reason for this is the uncertainty associated with pixel to pixel intensity fluctuations after laser sheet profile corrections. In our case this is found to be $\pm 8 \%$; this, in turn, corresponds to to an uncertainty of $\pm 11 \%$ in mixture fraction scale. Previous OH-LIF and numerical investigations conducted on the same experimental configuration have shown that ignition always occured at a region close to the jet tip along the axis rather than at the sides [2]. This can attributed to the disparate mixing behaviour as can be seen in Figure 1.

\section{Conclusions}

Ignition of hydrogen/air mixtures by jets of hot burned gases is studied using instantaneous, quantitative, two dimensional and temporally resolved mixture fraction plots. A novel method based on combining NO LIF measurements with numerical simulations of the underlying physical and chemical processes behind hydrogen/air ignition by hot jets and spectroscopic modelling of LIF of seeded NO is used for the mixture fraction measurements. The numerical simulations deliver the set of state variables (species concentration, temperature) accessed by the system during the interaction of hot burned hydrogen/air gases with unburned hydrogen/air mixture. The information so obtained is used for the spectroscopic simulation of NO-LIF signal in order to identify excitation schemes that deliver a sharp correlation to the desired state variable - mixture fraction. This correlation should also allow a unique determination of mixture fraction from the measured signal. Such an excitation scheme is used for the 2D mixture fraction measurements in the current study. The measured LIF-signals are then converted back into mixture fraction plots using the correlations.

The current study focusses mainly on two experimental configurations, one without ignition in the second vessel and the other with ignition where the period of interest was the time instance just before ignition. In both cases the method employed successfully captures the axial monotonic decay of the jet fluid concentration and the typical axisymmetric radial profiles of jet flows. The mixture fraction plots also highlight dissimilar mixing behaviour at regions near to the jet tip and the lateral sides. This is particularly interesting as previous OH-PLIF experiments showed that ignition always occurred at regions closer to the jet tip rather than at the lateral shear layers [2].

\section{Acknowlegdements}

The authors are grateful to Wolfgang Bessler (University of Heidelberg) and Christof Schulz (University of Duisburg) for providing a copy of their program LIFSim.

\section{References}

[1] Maas U, Pope, SB (1992). Symplifying chemical kinetics: Intrinsic low dimensional manifolds in composition space. Combust. Flame 88:239

[2] Sadanandan R (2007). Detailed investigation of ignition by hot gas jets. Proc. Combust. Inst. 31:719.

[3] Klaus P, Warnatz J. (1995). A contribution towards a complete mechanism for the formation of NO in flames. In: Joint meeting of the Frech and German section of the Combustion Institute

[4] Bessler W (2003). A versatile modelling tool for nitric oxide LIF spectra. In: Proceedings of the Third Joint Meeting of the U.S. sections of the Combustion Institute D33: 1

[5] Warnatz J, Maas, U, Dibble RW (2006). Combustion. Springer Verlag (ISBN 3-540-25992-9) 